\title{
Acurácia* dos indicadores de risco do Programa de Defesa da Vida dos Lactentes em região do Estado de São Paulo, Brasil.
}

\author{
Accuracy of risk indicators of an Infant Life Defense Program in a region of $S$. \\ Paulo State, Brazil.
}

\author{
Davi Rumel*, David Capistrano da Costa Filho*, Sandra Aiello , Sérgio Mikio Koyama , Wilf \\ Rosenbaum
}

RUMEL, D. et al. Acurácia dos indicadores de risco do Programa de Defesa da Vida dos Lactentes em região do Estado de São Paulo, Brasil. Rev. Saúde públ., S. Paulo, 26: 6 - 11, 1992. O Programa de Desfesa da Vida dos Lactentes da Secretaria de Higiene e Saúde do Munícipio de Bauru tem um critério diagnóstico para a inclusão de recém-nascidos dentro do programa. Este critério é formado pela combinação de 11 indicadores clínicos e sociais de risco à mortalidade infantil de fácil obtenção no momento do parto. Decidiu-se propor um critério diagnóstico alternativo, a partir dos mesmos indicadores clínicos e sociais, com maior sensibilidade para a mesma proporção de crianças matriculadas no Programa. Os dados hospitalares foram coletados no período de 11 de maio de 1986 a 10 de novembro de 1987. A mortalidade compreende o período entre 7 dias e 6 meses, que é o período de seguimento das crianças pelo Programa. Calculou-se para cada indicador o risco relativo bruto numa análise univariada e o risco relativo ajustado pela técnica de regressão logística. Criou-se um sistema de pontuação baseado na somatória dos excessos de risco de cada indicador.

Descritores: Fatores de risco. Saúde infantil. Sensibilidade e especificidade (epidemiologia).

\section{Introdução}

A Secretaria de Higiene e Saúde do Munícipio de Bauru, cidade do interior do Estado de São Paulo, vem expandido sua rede básica de saúde e organizando programas de assistência à saúde baseados em critérios epidemiológicos, desde 1984.

A existência de uma rede de assistência à saúde com atendimento universal, regionalizado e hierarquizado, aliada à cobertura de água tratada e esgoto a $98 \%$ e $90 \%$ da população respectivamente

\footnotetext{
* O mesmo significado do termo "acurácia" neste trabalho também é atribuído ao termo "validade" em alguns estudos.

* Departamento de Epidemiologia da Faculdade de Saúde Pública da Universidade de São Paulo - São Paulo, SP Brasil.

*** Secretaria de Higiene e Saúde do Municipio de Bauru Bauru, SP - Brasil.

${ }^{\star \star \star \star *}$ Pós-Graduando do Instituto de Matemática e Estatística da Universidade de São Paulo, SP - Brasil.

${ }^{\star \star \star \star}$ Computing Science Department, Simon Fraser University - Bumaby, B.C. - Canada.

Separatas/Reprints: D. Rumel - Av. Dr. Amaldo, 715 - 01255

- São Paulo, SP - Brasil.

Publicação financiada pela FAPESP. Processo Saúde Coletiva 91/4994-0.
}

(dados de 1986), além do baixo índice de desemprego e sub-moradia, fazem de Bauru uma cidade privilegiada para o aprimoramento dos serviços prestados pelo sistema de saúde.

$\mathrm{Na}$ área de assistência à criança há dois programas cuja seleção é baseada no enfoque de risco $^{2}$ : Programa de Defesa da Vida dos Lactentes, para crianças de 10 dias a 6 meses, e Programa de Controle de Desnutridos, para crianças de 7 meses a 2 anos ${ }^{3}$.

O Programa de Defesa da Vida dos Lactentes (PDVL) inicia-se através de visita hospitalar a todas as puérperas que deram à luz na cidade, realizada por auxiliares de enfermagem previamente treinadas para execução de tal função. A visita hospitalar envolve as seguintes atividades: a) entrevista onde são colhidas informações padronizadas a serem utilizadas para a determinação de eventual inclusão da criança no grupo de risco do programa; b) agendamento da primeira consulta médica para as crianças de risco na unidade de saúde mais próxima da residência ou preferência da mãe; c) trabalho educativo para o aleitamento materno e cuidados do recém-nascidos; e d) entrega de uma carta de parabenização pelo nascimento da parte do Prefeito. 
O que diferencia uma criança inscrita no Programa de uma não inscrita é que a responsabiliade pela saúde da primeira passa a ser também do serviço e não só da famılia. A assistência médica é a mesma em ambos os grupos. Porém, os inscritos são objeto de uma vigilância mensal por parte da enfermeira da unidade básica de saúde. Se uma criança do programa não estiver agendada para um retorno médico, ela é automaticamente agendada para um atendimento de enfermagem. Caso haja falta ou uma necessidade detectada, há uma visita domiciliar por parte de uma assistente social. $O$ incentivo ao aleitamento materno é prioritário neste acompanhamento.

Os fatores e/ou indicadores de risco adotados, basearam-se em revisão e no estudo dos óbitos infantis realizados pela Comissão de Investigação e Análise de Óbitos da Secretaria de Higiene e Saúde do Município de Bauru'. A exposição a um indicador de maior risco ou a dois de menor risco constituiu o critério diagnóstico adotado pelo PDVL, para selecionar uma criança ao grupo de risco. Este critério é detalhado a seguir.

\section{Indicadores isolados (de maior risco):}

- peso ao nascer inferior a $2.500 \mathrm{~g}$

- prematuridade

- gravidez manifestamente indesejada

- malformação congênita

\section{Indicadores simultâneos (de menor risco):}

- renda familiar per capita mensal igual ou menor que $1 / 4$ do salário mínimo

- mấe sem companheiro

- recém-nascido com 2 ou mais irmãos menores de 4 anos

- mãe analfabeta

- mãe com menos de 3 consultas de pré-natal na gravidez

- desemprego do chefe da familia

- idade materna inferior a 18 anos

O enfoque de risco pretende atuar no período patogênico da doença, abaixo do horizonte clínico, na fase de diagnóstico precoce, conforme diagrama de história natural das doenças de Leavell e Clark?.

O enfoque de risco visa à prevenção em nível individual antes que apareça a doença. Assim, os programas de saúde infantil, à base de critérios de risco, propõem um seguimento especial às crianças de maior probalidade de adoecer e ir a óbito, detectadas no momento em que o teste diagnóstico é aplicado, no caso, as entrevistas com as mães nas maternidades.
"A mágica da estatística não deveria obscurecer o valor do diagnóstico clínico e do prognósti$\mathrm{co}^{\prime \prime}$ - Lesinsky ${ }^{10}$. O prognóstico clínico, por ser dinâmico, é sempre melhor que o resultado de um teste aplicado num determinado momento. A probalidade de adoecer ou ir a óbito muda a cada momento conforme novos fatores de risco se somam à história de vida da criança ou à evolução clínica da mesma.

Um programa de saúde infantil, à base de critérios de risco identificados no momento do nascimento, não exclui a participação do clínico e demais profissionais de saúde no diagnóstico de patologias ou detecção de outros fatores de risco presentes após o nascimento, na população de crianças não pertecentes ao grupo de risco. Assim, o diagnóstico de uma patologia de curso crônico ou a identificação de um desmame precoce, em crianças não diagnosticadas como de risco no momento do nascimento, podem ser critérios de inclusão no programa de assistência especial a estas crianças.

Estes fatos (que o prognóstico clínico é melhor e que o grupo de risco pode ser ampliado após o nascimento) não invalidam a importância do diagnóstico de risco no momento do nascimento, já que nem todas as crianças têm acesso à assistência médica por razões econômicas da familia, culturais e psicológicas da mãe, e deficiências de oferta por parte dos serviços públicos. A garantia de contatos periódicos com unidades básicas de saúde, visita domiciliar e assistência social quando necessária, podem prevenir a doença, detectá-la precocemente e dar o devido suporte às orientaçōes terapêuticas.

Um programa deste tipo não é, e nem se propõe a ser, uma abordagem de prevenção primária, coletiva, à mortalidade infantil. Outras abordagens, que visam a prevenir riscos inaceitáveis ao padrão de desenvolvimento atual, são de igual ou maior importância como: saneamento básico, atividades de incentivo ao aleitamento materno, acesso a serviços ambulatoriais de saúde, recursos hospitalares para gestantes de alto risco e neonatos com baixò peso.

A partir da análise dos dados provenientes do PDVL e dos cartórios da cidade, o presente trabalho procura propor um critério diagnóstico de maior sensibilidade para a mesma proporção de crianças selecionadas pelo PDVL.

No presente estudo, sensibilidade é a proporção das crianças que foram a óbito e estavam inscritas no Programa em relação ao total das crianças que foram a óbito. Não foi considerada a especifícidade dos critérios diagnósticos, pois o objetivo é a minimização da possibilidade de se ter falsos negativos independente da proporção de falsos positivos que venham a fazer parte do Programa. 


\section{Material e Método}

\section{População de estudo e delineamento}

Por ser o Município de Bauru, Estado de São Paulo, um centro de invasão de assistência hospitalar e óbitos, é uma cidade propícia a estudos epidemiológicos de coorte com curto período de seguimento, pois as perdas são pequenas comparadas a cidades em que há evasão por assistência médica. Em 1987, dos 92 óbitos infantis de crianças nascidas e residentes em Bauru, 90 ocorreram no Município (Fundação SEADE).

A populacão de estudo são todas as crianças que nasceram em Bauru entre 11 de maio de 1986, data do início do Programa, e 10 de novembro de 1987, num total de 6.989 crianças, já excluídas as crianças não residentes naquele município $\mathrm{e}$ as nascidas de parto múltiplo. As criancas foram seguidas até 6 meses de idade inclusive, pois $95 \%$ dos óbitos infantis em Bauru ocorrem neste periodo, e é este o período de abrangência do PDVL.

Das 6.989 crianças, cujos dados foram analisados, 66 morreram na primeira semana e 45 entre o oitavo dia e o fím do sexto mês.

A coleta de dados de óbitos se estendeu até 10 de junho de 1988. Os óbitos foram coletados nos três cartórios da cidade.

\section{Procedimentos}

A coleta de informações envolveu duas fontes: fichas de primeiro atendimento preenchidas pelos pediatras (para as variáveis prematuridade, malformação congênita e peso ao nascer) e entrevistas com as mães (demais variáveis). As informações colhidas das fichas clínicas preenchidas por inúmeros médicos que não foram treinados quanto aos critérios para seu preenchimento podem sugerir um vício de aferição. Assim, por exemplo, somente os casos graves de malformação congênita podem ter sido registrados.

$O$ indicador de risco renda familiar foi determinado num primeiro momento como de $1 / 3$ do salário mínimo per capita. Como o número de criancas inscritas superou a capacidade para visitas domiciliares, logo no início do Programa, este limite caiu para $1 / 4$, que é o limite adotado por este estudo.

O método de análise foi o de análise multivariada por regressão logística por ser a variável dependente (efeito) categórica e as variáveis independentes (prováveis fatores de risco) não tão indenpendentes assim, ou seja, além de associadas com o efeito, estas variáveis podem estar associadas entre si sendo que uma delas, identificada

\footnotetext{
Comunicação pessoal
}

como fator de risco numa análise univariada, pode deixar de sê-lo já que seu impacto sobre o efeito provém da associação com outro fator de risco ("confounding") ${ }^{8}$. Outro aspecto da análise multivariada por regressão logística é que ela permite a investigação de interações entre as variáveis indepentes ${ }^{\top}$.

Alguns indicadores de risco não foram utilizados ao longo de todo o estudo pelos motivos que seguem. A gravidez manifestamente indesejada foi introduzida para identificar uma possível rejeição materna, hipótese aventada como indicador de risco pelos estudos da Comissão de Investigação e Análise de Óbitos. Buscava-se saber se a gravidez havia sido planejada e, nos casos negativos, se após seu conhecimento ela foi ou não aceita. Este indicador de risco foi tabulado somente para os primeiros 6 meses do programa, pois a partir de então os dados foram redirecionados por uma abordagem com ênfase à rejeição pós-nascimento da criança.

Os indicadores de risco, mãe analfabeta e mãe sem companheiro, foram coletados, até completarem um ano de Programa, ocasião em que foi alterado o critério de inclusão das crianças.

Esta ausência de informação prejudicou a análise de regressão logística. Se fosse analisada toda a coorte ter-se-ia que eliminar três importantes variáveis. Para utilizar todas elas e assegurar o menor erro possível, foram identificados os coeficientes da equação de regressão logística com os $2 / 3$ iniciais da coorte eliminando os dados da variável gravidez indesejada. Repetiu-se a análise com o $1 / 3$ inicial da coorte para identificar o coeficiente da variável gravidez indesejada.

Outro fato que prejudicou a análise de regressão logística, é que o indicador malformação congênita está tão associado com o efeito (mortalidade) que não foi possível a análise dos dados na presença desta variável. Foi arbitrado um alto coeficiente a este indicador, suficiente para colocar as poucas crianças, com este indicador, no Programa na menor proporção de criancas selecionadas consideradas no presente estudo.

Algumas transformações de variáveis quantitativas foram realizadas na análise de regressão logística. A distribuição do risco relativo por faixa de peso é uma curva do tipo $y=1 / x$. A distribuição do risco relativo por faixa de idade materna é uma curva do tipo $y=(x-k)^{2}$, sendo $k=27$, ponto médio da faixa de menor risco relativo. Estas variáveis foram transformadas obedecendo estas equaçōes 5 .

Para identificar um critério diagnóstico alternativo utilizamos dois sistemas de pontuação com base nos coeficientes calculados pelas regressões logísticas. Foram realizadas duas regressões: uma com variáveis quantitativas e qualitativas e outra 
com as variáveis quantitativas categorizadas, ou seja, todas as variáveis se apresentando na forma qualitativa.

A partir dos coeficientes, calcularam-se estimativas de riscos relativos que deram origem a excessos de risco ajustados, número utilizado no sistema de pontuação proposto para o critério diagnóstico alternativo.

O excesso de risco é o valor do risco relativo subtraido de uma unidade $(R R-1)^{4}$. Julgamos que o uso do excesso de risco era o mais adequado para o propósito de somar diferentes indicadores em um sistema de pontuação.

Por exemplo: uma criança tem exposição a dois fatores de risco, $A$ e $B$, com $R_{A}$ igual a 1,9 e $\mathrm{R}_{\mathrm{B}}$ igual a $1,5 \mathrm{e}$, outra, a três fatores de risco, $\mathrm{C}$, $D$ e $E$ com $R_{C}$ igual a $1.3, R_{D}$ igual a 1,4 e $R_{E}$ igual a 1,6. Somando os riscos relativos, a primeira criança tem uma pontuação menor $(3,4)$ que a segunda criança $(4,3)$. Somando os excessos de risco ocorre o contrário, a primeira criança tem uma pontuação maior $(1,4)$ que a segunda $(1,3)$. Os excessos de risco permitem eliminar o valor 1 , que significa ausência de risco, e que aparece devido ao risco relativo ser o quociente de uma divisão.

Optou-se por um sistema de pontuação aditivo em vez de multiplicativo por ser o modelo aditivo mais adequado para o estudo de interação de fatores de risco 11 .

No PDVL, a presença de cada indicador de risco isolado equivale a um ponto e cada risco simultâneo a 0,5 ponto, com um corte de um ponto no mínimo para cada criança pertencer ao grupo de risco.

Finda a identificação do número de pontos de cada criança, em sistema de pontuação, foram comparados os diferentes sistemas quanto a melhor combinação entre sensibilidade e proporção de crianças no grupo de risco.

O PDVL atuou somente na prevenção da mortalidade e não na prevenção de fatores de risco detectados no momento do nascimento. A sua existência, na fase de coleta de dados, pode ter diminuido a intensidade dos riscos associados aos indicadores utilizados pelo Programa, mas não deixando de identificá-los. Se esta interferência foi igual para todos os indicadores, a sensibilidade não foi afetada.

\section{Resultados e Discussão}

A análise de regressão logística com variáveis quantitativas apresentou os seguintes resultados:

Probalidade de morte $=1 /(1+\exp -(-7,618$ $+0,4280 x_{1}+6512 / x_{2}+1,061 x_{3}+0,001699\left(x_{4}-\right.$ $27)^{2}+0,1666 \times_{5}+1,014 \times 6+0,6345 \times 7-0,04197 x_{8}$ $\left.+0,6039 x_{9}-0,04587 x_{10}\right)$ )
Onde $\mathrm{x}_{1}=$ prematuro; $\mathrm{x}_{2}=$ peso ao nascer; $\mathrm{x}_{3}=$ gravidez indesejada; $\mathrm{x}_{4}=$ idade materna; $\mathrm{x}_{5}$ $=$ mãe analfabeta; $x_{6}=$ mãe sem companheiro; $x_{7}$ $=$ chefe de familia desempregado; $\mathrm{x}_{\mathbf{8}}=$ renda $\mathrm{fa}$ miliar per capita; $x_{9}=$ dois ou mais irmãos menores de 4 anos; $x_{10}=$ número de consultas de prénatal.

A Tabela 1 apresenta os resultados da análise de regressão logística com variáveis qualitativas, expressos pelos riscos relativos ajustados. São apresentados os riscos relativos brutos provenientes da análise univariada para efeito de comparação.

A Tabela 1 indica que prematuridade apareceu na análise univariada como fator de risco provavelmente devido a sua associação com baixo peso. (Em termos biológicos, prematuridade é um dos determinantes de baixo peso, e é parte da rede de causalidade dentro de um raciocínio etiológico). Com renda abaixo de 0,25 salários mínimos per capita aconteceu o mesmo, provavelmente devido a sua associação com desemprego, dois ou mais irmãos menores de 4 anos, mãe analfabeta e mãe sem companheiro. Idade materna menor de 18 anos e menos de 3 consultas de pré-natal deixaram de ser riscos e se tornaram fatores de proteção. Isto não tem o mínimo senso em termos biológicos. Estes resultados podem ser explicados pela falta de significância destes coeficientes e, provavelmente, pelos pontos de corte destas variáveis não terem sido adequados. $\mathrm{Na}$ análise multivariada com variáveis quantitativas, os extremos de idade materna (adolescentes e acima de 40 anos) são relevantes para a probalidade de morte. Porém, o número de consultas de pré-natal parece ser indenpendente de mortalidade infantil neste período de estudo (acima de 7 dias de vida). Tendo em vista o exposto acima, não foram consideradas estas va-

Tabela 1. Riscos relativos brutos e ajustados por in dicador de risco em relação à mortalidade entre 7 dias $\theta 6$ meses de idade.

\begin{tabular}{lcc}
\hline Indicador de risco & RR bruto & RR ajustado \\
\hline Prematuridade & $5,34^{*}$ & 1,80 \\
Peso < $2.500 \mathrm{~g}$ & $4,86^{*}$ & $4,81^{\circ}$ \\
Gravidez indesejada & $3,66^{\circ}$ & 3,52 \\
Malformação congênita & $71,86^{*}$ & - \\
Mãe < 18 anos & 1,56 & 0,55 \\
Mãe analfabeta & 1,62 & 1,65 \\
Mãe sem companheiro & $3,53^{*}$ & $3,30^{*}$ \\
Desemprego & 2,28 & 2,12 \\
Renda <=0,25 S.M. p/c & $3,27^{*}$ & 1,23 \\
2 ou + irmãos <4 anos & 2,07 & 1,90 \\
$<3$ cons. pré-natal & 1,51 & 0,50 \\
\hline p < 0,05 & &
\end{tabular}


riáveis no sistema de pontuação, com base nos resultados da análise de regressão logística com todas as variáveis categorizadas (arbitrou-se risco relativo ajustado igual a um).

Devido ao pequeno número de óbitos, a interação entre dois indicadores não pode ser testada ${ }^{6}$.

A partir destes resultados, construiu-se uma tabela de pontos entre as diversas alternativas metodológicas, transformando os riscos relativos em excessos de risco, de acordo com a argumentação expressa anteriormente na parte "procedimentos" e apresentando o sistema de pontos implícito na proposta do PDVL. Na Tabela 2 não foram apresentados os pontos provenientes da análise de regressão logística com variáveis quantitativas, pois cada valor de uma variável quantitativa corresponde a uma diferente pontuação.

Tabela 2. Excessos de risco (ER) por indicadores de risco em relação a mortalidade entre 7 dias e 6 meses de idade.

\begin{tabular}{lcc}
\hline Critérios de risco & ER/ajust. & PDVL \\
\hline Prematuridade & 0,8 & 1,0 \\
Peso < $2.500 \mathrm{~g}$ & 3,8 & 1,0 \\
Gravidez indesejada & 2,5 & 1,0 \\
Malformação congênita & $4,0^{\circ}$ & 1,0 \\
Mãe < 18 anos & 0 & 0,5 \\
Mãe analfabeta & 0,7 & 0,5 \\
Mãe sem companheiro & 2,3 & 0,5 \\
Desemprego & 1,1 & 0,5 \\
Renda <=0,25 S.M. p/c & 0,2 & 0,5 \\
2 ou + irmãos <4 anos & 0,9 & 0,5 \\
$<3$ cons. pré-natal & 0 & 0,5 \\
\hline
\end{tabular}

- arbitrado

O critério diagnóstico adotado pelo PDVL discriminou $16 \%$ dos recém-nascidos para recebimento de uma atenção especial com uma sensibilidade de 58\%. A Tabela 3 apresenta os resultados do seguimento destas crianças quanto a mortalidade.

Tabela 3. Número e percentual de criancas nascidas e residentes em Bauru entre 11/05/86 e 10/11/87, segundo critérios de inclusão no Programa de Defesa da Vida dos Lactentes e mortalidade entre 6 dias e 7 meses.

\begin{tabular}{cccc}
\hline PDVL & Óbitos & Sobreviventes & Total \\
\hline Sim & $26(58 \%)$ & $1.081(16 \%)$ & $1.107(16 \%)$ \\
Nâo & $19(42 \%)$ & $5.797(84 \%)$ & $5.816(84 \%)$ \\
\hline Total & $45(100 \%)$ & $6.878(100 \%)$ & $6.923(100 \%)$ \\
\hline
\end{tabular}

As Tabelas 4 e 5 apresentam os resultados quanto à sensibilidade e proporção de crianças classificadas como de risco de acordo com os diversos sistemas de pontuação. Para a análise com 10
Tabela 4. Sensibilidade e proporção de crianças classificadas como de risco de acordo com análise de re. gressão logística com variáveis quatitativas

\begin{tabular}{ccc}
\hline $\begin{array}{c}\text { Limite de } \\
\text { probabilidade } \\
\text { de morte }\end{array}$ & Sensibilidade & $\begin{array}{c}\text { Proporção de } \\
\text { crianças }\end{array}$ \\
\hline$>0,010$ & $53 \%$ & $8 \%$ \\
$>0,008$ & $58 \%$ & $12 \%$ \\
$>0,0075$ & $58 \%$ & $14 \%$ \\
$>0,007$ & $64 \%$ & $15 \%$ \\
$>0,0065$ & $67 \%$ & $17 \%$ \\
$>0,006$ & $69 \%$ & $20 \%$ \\
$>0,005$ & $71 \%$ & $26 \%$ \\
\hline
\end{tabular}

Tabela 5. Sensibilidade e proporçāo de crianças clas. sificadas como de risco de acordo com a análise de regressảo logística com variáveis qualitativas $\theta \mathrm{com} 0$ sistema de pontuação do Programa de Defesa da Vida dos Lactentes

\begin{tabular}{ccccc}
\hline $\begin{array}{c}\text { Ponto de } \\
\text { corte }\end{array}$ & $\begin{array}{c}\text { Análise multivar. } \\
\text { qualitativa } \\
\text { Sens. }\end{array}$ & crianças & Sens. & PDVL crianças \\
\hline$>$ ou $=3,0$ & $51 \%$ & $7 \%$ &. &. \\
$>$ ou $=2,5$ & $53 \%$ & $10 \%$ &. &. \\
$>$ ou $=2,3$ & $62 \%$ & $13 \%$ &. &. \\
$>$ ou $=1,1$ & $62 \%$ & $16 \%$ & $58 \%$ & $16 \%$ \\
$>$ ou $=0,5$ & $64 \%$ & $20 \%$ & $80 \%$ & $32 \%$ \\
\hline
\end{tabular}

variáveis quantitativas os pontos equivalem aos limites de probalidade de morte.

Como pode-se observar, o PDVL segue $16 \%$ das crianças vivas após o sétimo dia de idade com $58 \%$ de sensibilidade. Se for mantida a mesma proporção de crianças, o melhor critério seria utilizar a equação de regressão logística com variáveis quantitativas no. limite de probalidade, 6,5 óbitos por mil criancas. Teriamos $17 \%$ de crianças selecionadas com $67 \%$ de sensibilidade.

\section{Comentários Finais}

Partindo do pressuposto que este Programa é eficaz e efetivo, deve-se mantê-lo e adotar o sistema de pontuação proposto por este estudo.

As informaçōes que permitiram esta análise não devem ter sua coleta interrompida, pois no futuro, com um número de óbitos maior, será possivel a elaboração de uma equação de regressão logística com resultados mais significantes que possibilite um processo de seleção ainda mais adequado.

O cálculo de todas as sensibilidades e proporção de crianças em risco estāo subestimados pela ausência de informação sobre gravidez indesejada, 
em $2 / 3$ da coorte e escolaridade e mãe sem companheiro em $1 / 3$ da coorte. Caso estas variáveis tivessem sido coletadas em toda a coorte, o número de crianças classificadas como de risco seria maior, e o número de óbitos no grupo de risco também seria maior uma vez que estas variáveis são bastante preditoras de óbito infantil como este estudo demonstrou. Portanto, o ganho em sensibilidade, comparando o PDVL com os sistema de pontuação proposto, deve ser ainda maior que o apresentado nos resultados do presente trabalho.

RUMEL, D. et al. [Accuracy of risk indicators for an Infant Life Defense Program in a region of $S$. Paulo State, Brasil]. Rev. Saúde públ., S. Paulo, 26: 6 - 11, 1992. The Infant Life Defense Program of Health Department of Bauru City SP, Brazil, has diagnostic criteria for the inclusion of newborns. The diagnostic criteria combine eleven social and clinical indices of infant mortality risk, defined by in the light of a review of the literature and previous case studies. The indices are easily collected at the hospitals during delivery. The objective of this study is to propose an alternative diagnostic criteria, using the same social and clinical indices, of greater sensitivity and same proportion of children included in the program. The data on the newborns were collected between May 11, 1986 and November 10, 1987. The mortality period was defined as between 7 days and 6 months, which was the follow-up period for the infants enrolled in the program. The method of analysis was the determination for each index of the crude relative risk in a univariate analysis and the adjusted relative risk using the logistic regression procedure. A score system was constructed on the basis of the sum of the excess risk of each index.

Keywords: Risk factors. Child health. Sensitivity and specificity (epidemiology)

\section{Referências Bibliográficas}

1. AIELLO, S.; RUMEL, D.; FRIGÉRIO, M.; PIMENTA, A. Comissão de investigação e análise de óbito. In: Capistrano D. Saúde para todos: um desafio ao município. São Paulo, Hucitec-Oboré, 1985. p. 55-73.
2. BACKETT, E.M.; DAVIES, A.M.; BARVAZIAN, A.P. Introduçăo. In: Backett, E.M.; Davies, A.M.; Barvazian, A.P. $O$ enfoque de risco na assistência a sacide, com especial referência a saúde materno-infantil, inclusive planejamento familiar. Washington DC, Organização Panamericana da Saúde, 1985. p. 1-6 (OPAS - Publicação Científica, 491).

3. CAPISTRANO, D.; RUMEL, D.; PIMENTA, A.; MELHADO, LA. Vencendo a mortalidade infantil. In: Capistrano, D. \& Pimenta, A. Saúde para todos: um desafio ao município. $2^{\prime}$ ed. São Paulo, Hucitec, 1988. p. $115-30$.

4. COLE, P. \& MACMAHON, B. Attibutable risk percent in case-control studies. Brit. J. prev. soc. Med., 25: $242-4,1971$.

5. HOSMER, D.W. \& LEMESHOW, S. Model-building strategies. In: Hosmer, D.W. \& Lemeshow, S. Applied logistic regression. New York, John Wiley \& Sons, 1989. p. 82-134.

6. KELSEY, J.L; THOMPSON, W.D.; EVANS, A.S. Measurement II. In: Kelsey, J.L.; Thompson, W.D.; Evans, A.S. Methods in observational epidemiology. New York, Oxford University Press, 1986. p. 337-51.

7. KLEINBAUM, D.; KUPPER, L; MULLER, K. Regression diagnostics. In: Kleinbaum, D.; Kupper, L.; Muller, K. Apllied regression analysis and other multivariable methods. 2nd ed. Boston, PWS-KENT Publishing Company, 1988. p. 181-225.

8. KLEINBAUM, D.; KUPPER, L; MORGENSTERN, H. MOdeling: analysis strategy. In: Kleinbaum, D.; Kupper, L; Morgenstem, H. Epidemiologic researh. Belmont, C.A., Lifetime Leaming Publication, 1982. p. 447-56.

9. LEAVELL, H.R \& CLARK, E.G. Niveis de aplicação da medicina preventiva. In: Leavell, H.R. \& Clark, E.G. Medicina preventiva. São Paulo, Ed. McGraw Hill do Brasil, 1976. p. 11-36.

10. LESINSKY, J.S. High-risk pregnancy: unresolved problems of screening, management, and prognosis. Obstet. Gynec. 46: 599-603, 1975.

11. ROTHMAN, KJ. Interactions between causes. In: Rothman K.J. Modern epidemiology. Boston, Little, Brown and Company, 1986. p. 311-26. 